RESEARCH ARTICLE

\title{
Effectiveness of Blood Pressure and Body Temperature Screening for Severity in COVID-19 Patients
}

\author{
Sushma Verma ${ }^{\star 1}$, Mohammad Abbas ${ }^{\star 1,2}$, Shrikant Verma ${ }^{1}$, Almas Khan ${ }^{1}$, Aliya Abbas Rizvi ${ }^{1}$, Syed \\ Tasleem Raza ${ }^{3}$, Farzana Mahdi ${ }^{4}$
}

*Sushma Verma and Mohammad Abbas contributed equally to this work as first authors

\author{
'Department of Personalized and Molecular Medicine, Era University, Lucknow, Uttar Pradesh, India \\ ${ }^{2}$ Department of Microbiology, Era University, Lucknow,-Uttar Pradesh, India \\ ${ }^{3}$ Department of Biochemistry, Eras Lucknow Medical College and Hospital, Era University, Lucknow, Uttar Pradesh, \\ India \\ ${ }^{4}$ Department of Medicine, Eras Lucknow Medical College, and Hospital, Era University, Lucknow, Uttar Pradesh, India
}

\begin{abstract}
Objectives: Coronavirus disease-2019 (COVID-2019) cases should be classified as severe and mild cases in COVID-19 for additional care requirements to decrease the mortality rate of the covid-19 infected patients. The study aims to investigate whether blood pressure, temperature, and gender are linked with the severity of COVID-19.
\end{abstract}

Methods: This study incorporated $n=495$ COVID-19 patients, whereas 195 cases were excluded from the study due to insufficient data. In total, 300 cases were analyzed, of which 205 patients are from the mild category and 95 from the severe category.

Results: We observed that initial systolic blood pressure was significantly high, although diastolic blood pressure significantly decreases in severity cases compared to mild COVID-19 cases. Furthermore, we observed that in severe COVID-19 instances, initial body temperature was substantially higher than in moderate cases. We also observed that male cases are more prone to have severity as compared to female cases.

Conclusion: This research will aid in the early detection of the severity of COVID-19 cases and nonseverity, which is critical in the current situation. J Microbiol Infect Dis 2021; 11(3):147-151.

Keywords: COVID-19, Severity, Blood Pressure, Body Temperature, Gender

\section{INTRODUCTION}

SARS-CoV-2 (COVID-19), a coronavirus disease caused by the SARS-CoV-2 virus, is a potentially fatal disease that affects people worldwide [1]. Several COVID-19 patients had mild/severe fever, cough, and weakness, while severe instances had dyspnea. [2]. WHOChina Joint Mission on COVID-19 reported that $80 \%$ of the 55,924 patients had mild todirect illness, including both non-pneumonia and pneumonia cases, whereas $13.8 \%$ created severe sickness and $6.1 \%$ created to a basic stage requiring intensive care [3]. Early diagnosis of individuals at risk of developing a critical illness can aid in providing appropriate treatment and lowering the mortality rate. Mortality or Serious Condition (Death or Intermittent Mandatory Ventilation (IMV)) are two factors that statistically meaningful variations observed between the three $\mathrm{WHO}$ severity categories [4].

Critical illnesses of COVID-19, including signs such as acute respiratory distress syndrome (ARDS), acute respiratory impairment, coagulopathy, septic shock, and metabolic acidosis, have advanced quickly in intensive

Correspondence: Dr. Farzana Mahdi, Department of Personalized and Molecular Medicine, Era University, Lucknow, Uttar Pradesh, India 
care units [5-7]. COVID-19 intensity must be evaluated to assess the suitability of mitigating strategies and aid in preparation for healthcare needs as epidemics arise. Diabetes (10-20\%), hypertension (10-15\%), and other cardiovascular and cerebrovascular disorders (7-40\%) were the most common comorbidities reported by $20-51 \%$ of patients at the time of admission [8-10]. In unadjusted epidemiological research describing the characteristics of the COVID-19 pandemic in China, patients with COVID-19 and hypertension were found to have a greater risk of negative outcomes [11]. There is an overrepresentation of hypertension in hospitalized and critically ill COVID-19 patients show ever; it is unclear if this link is causal or masked by age and other comorbidities.

Notwithstanding these perceptions, the connection between hypertension as well as COVID-19 is not clear. The seriousness of COVID-19 sickness is slanted towards the older populace who has an elevated pervasiveness of hypertension. Fever is a process in which the body temperature increases and deviates from normal values $[12,13]$, and according to Saladin's outstanding scientific text (28), it is a helpful process as long as it does not persist [14]. A metaanalysis of 1995 COVID-19 cases from China found that $89 \%$ had a fever, with nearly half of them being febrile at the time of hospitalization [15]. Fever was also found in $64 \%$ of healthcare workers who tested positive in New York, $35 \%$ of imported COVID-19 cases in Taiwan, and $45 \%$ of patients with mildmoderate illness in Europe [15-17].

An exceptionally high number of cases is emphasized to find an accurate prognostic marker for effectively using the facilities and reducing the mortality rate. Early identification of patients at risk of developing the severe disease is essential, as it may assist in providing adequate treatment and maximizing the use of limited resources [4]. Fever and blood pressure were found as leading factors in a majority of COVID-19 cases [18]. So, we looked at whether blood pressure, temperature, or gender was linked to the severity level of COVID-19.

\section{METHODS}

This study was performed in Era's Lucknow Medical College and Hospital, Lucknow, India. Between August and October 2020, patients who tested positive for SARS-CoV-2 were eligible to take part in this study.

\section{Design}

All COVID-19 infected patients who presented to the hospital and were hospitalized to various wards were included in this retrospective cohort analysis. A real-time reverse transcription-polymerase chain reaction was used to test swab samples from the nose and throat [19]. At the admission time of the infected patients, data for the outcome variables of body temperature and blood pressure were retrieved. Fever was defined as a body temperature of $\geq 38{ }^{\circ} \mathrm{C}$, and blood pressure was classified as $120 / 80 \mathrm{~mm} \mathrm{Hg}$, according to Indian Government Rules.

\section{Data collection}

A total number of confirmed cases $(n=495)$ were recruited, and in which 195 cases were excluded due to missing data. We included 300 COVID-19 infected patients in our study, in which 205 patients are from the mild category and 95 from the severe category. Patients' clinical data (blood pressure, temperature) during hospitalization were collected. All data were double-checked before being input into the computer database. (Figure 1).

\section{Analysis}

All analysis was done by prism software (version V). Each group's continuous variables will be summarized as mean $\pm S E$ and compared using the Student's t-test. For $\mathrm{p}<0.05$, all $\mathrm{p}$ values were considered statistically significant.

\section{RESULTS}

Four hundred ninety-five patients with COVID19 were evaluated, and 300 of them were included in the study. Out of them 196 were males $(65.3 \%)$, and 104 were females $(34.7 \%)$. According to their clinical presentation, 95 of them $(31.7 \%)$ were severe patients. The proportion of the severe cases were similar among males $(n=65,33.2 \%)$ and females $(n=30,28.9 \%)(p<0.05)$. The blood pressures of the patients showed significant variations according to their severity status (Figure 2). We found high systolic blood pressure in severe cases compared to mild cases (mean $131.2 \mathrm{mmHg}$ vs. $126.1 \mathrm{mmHg}$, respectively; $p=0.02$ ), whereas diastolic blood pressure was found significantly low in severity 
cases $(79,8 \mathrm{mmHg}$ vs. $84,8 \mathrm{mmHg}$ respectively; $p<0.001)$. On the other hand, this study revealed that the initial body temperature of severe COVID-19 cases was significantly high compared to mild cases $(p=0.02)$ (Figure
$3)$. In addition, seven of the severe cases (8\%) died in severe cases, of which 5 were males. The mortality rates were similar in males $(7.7 \%$ vs. $6.7 \%$ ) and females 1 ).

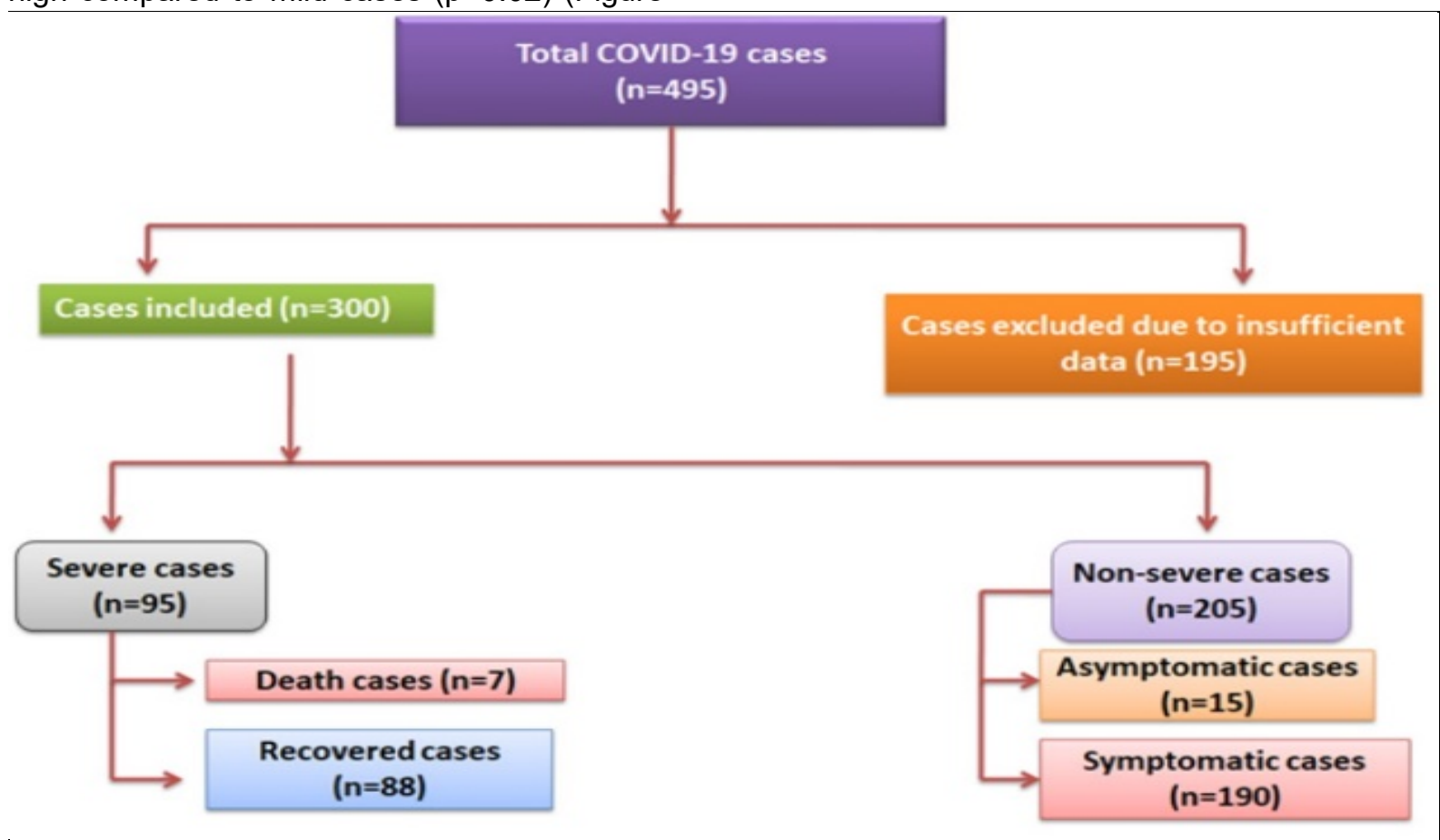

Figure 1. Flowchart showing outlining of patients' selection of COVID 19 for this study.

Table 1. Association of blood pressure and temperature with COVID-19 severity and gender vies.

\begin{tabular}{|c|c|c|c|}
\hline Patients & Mild $(n=205)$ & Severe $(n=95)$ & $P$ value \\
\hline Systolic Blood Pressure (mmHg) & $126.1 \pm 1.36$ & $131.2 \pm 1.91$ & 0.021 \\
\hline Diastolic Blood Pressure, (mmHg) & $84.83 \pm 0.82$ & $79.77 \pm 1.40$ & $<0.001$ \\
\hline Temperature $\left({ }^{\circ} \mathrm{C}\right)$ & $95.6 \pm 0.18$ & $96.05 \pm 0.30$ & 0.026 \\
\hline Female Patients $(n=104)$ & Mild $(n=74)$ & Severe $(n=30)$ & \\
\hline Systolic Blood Pressure, $(\mathrm{mmHg})$ & $123.1 \pm 2.80$ & $135.2 \pm 3.86$ & 0.008 \\
\hline \multirow[t]{2}{*}{ Diastolic Blood Pressure, (mmHg) } & $84.32 \pm 1.13$ & 82.37 & 0.305 \\
\hline & & \pm 2.48 & \\
\hline Temperature $\left({ }^{0} \mathrm{C}\right)$ & $95.74 \pm 0.28$ & $96.52 \pm 0.41$ & 0.077 \\
\hline Male Patients $(n=196)$ & Mild $(n=131)$ & Severe $(n=65)$ & \\
\hline Systolic Blood Pressure, (mmHg) & $127.7 \pm 1.41$ & $129.1 \pm 2.15$ & 0.499 \\
\hline Diastolic Blood Pressure, (mmHg) & $85.12 \pm 1.11$ & $78.4 \pm 1.71$ & $<0.001$ \\
\hline Temperature $\left({ }^{0} \mathrm{C}\right)$ & $95.5 \pm 0.24$ & $95.8 \pm 0.40$ & 0.135 \\
\hline
\end{tabular}

*Bold numerals provided in the table show association with disease. Data are represented as Mean \pm SE.

DISCUSSION

The current study found that body temperature, blood pressure, and gender substantially influenced COVID-19 severity. Patients with COVID-19 and hypertension have been related to an enlarged risk of unfavorable outcomes [20]. In comparison to healthier individuals, persistent hypertension was more common among those who died, according to Chen et al. [20]. In addition, a study revealed that hypertensive COVID-19 cases showed more dependency on intensive care units than other COVID-19 cases [16]. 


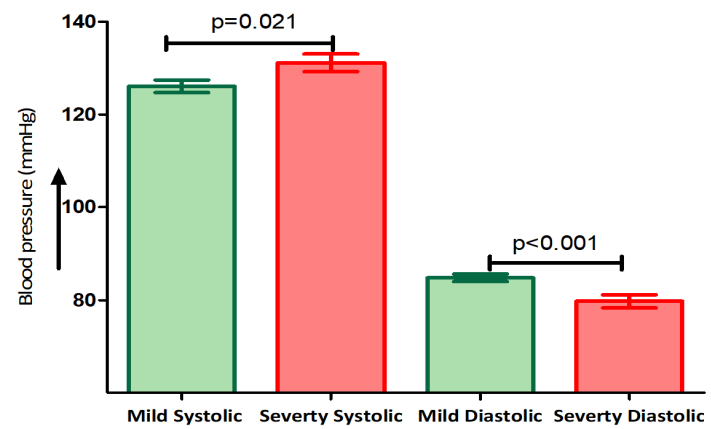

Figure 2. Elevated Blood pressure in 300 Covid-19 cases $\left({ }^{*} p\right.$ value $\left.<0.05\right)$.

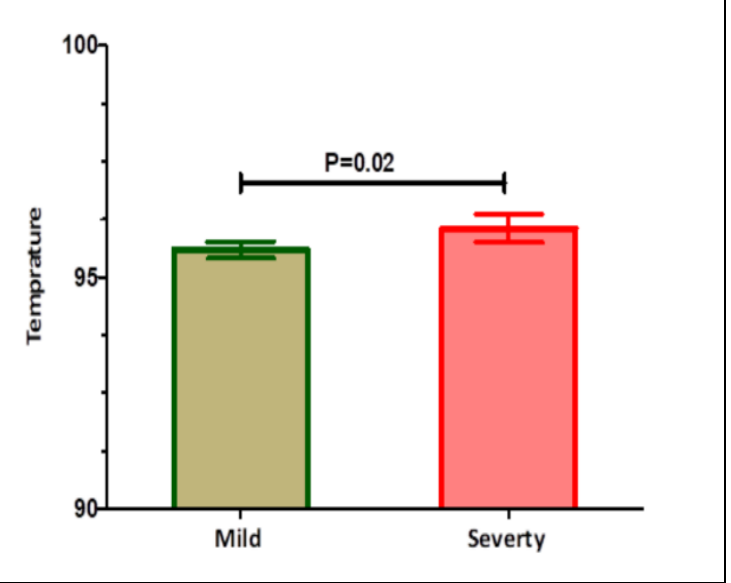

Figure 3. Body temperature variation in the cases.

Few studies indicated that the use of RAAS inhibitors in cases with COVID-19 versus other antihypertensive drugs might be act as protective against complications and death [19]. The Renin-angiotensin-aldosterone system (RAAS), activated by the angiotensinconverting enzyme II (ACE-2) receptor, acts as a binding site for SARS-CoV-2. In the USA, approximately $50 \%$ of cases are prescribed Aldosterone Receptor Blockers (ARBs), angiotensin-converting enzyme inhibitors (ACE-1), and aldosterone antagonists [21]. Compared to non-users of ACE-1s or ARBs, retrospective research of 1,128 hypertension subjects indicated a minor threat of all-cause mortality [19]. In this study, we found that systolic blood pressure significantly raised in severe cases compared to mild cases, whereas diastolic blood pressure significantly decreased in severe cases.

In this study, we also analyzed the body temperature of 300 COVID-19 cases and assed the patients with a high body temperature at the time of admission were more vulnerable to severity than others. Tharakan et al. show that one out of three patients arriving at a most intense BT above
$39.5{ }^{\circ} \mathrm{C}$ died [22]. Bielecki discovered that a low-temperature cut-off estimation of $37.1^{\circ} \mathrm{C}$ will miss over $33 \%$ of suggestive COVID-19 instances upon arrival of determination, resulting in a large number of false positives [23]. Mitra et al. also reported that the affectability of fever likewise shows up even lower in the underlying phases of the sickness versus later throughout the disease [15]. Thus, it is still unclear whether lowering the temperature in COVID-19 patients who are critically ill will minimize the inflammatory response and enhance their outcome compared to other COVID-19 patients. Future examinations are essential to address this inquiry. In COVID-19 [7], fundamental inflammation is associated with acute respiratory distress syndrome, which is linked to a high fatality rate of up to $32.5 \%$ [24].

The present study suggested that the female cases showed less severity as compared to male cases. Males tend to develop $68.42 \%$ high severity as compared to female (31.58\%) COVID-19 cases. Similarly, several studies observed a high percentage of male cases COVID-19 cases $[25,26]$. In our study, male individuals showed $5 \%$ mortality in the severe group, whereas females showed $2 \%$ mortality. As a result, regardless of age or susceptibility, gender is a risk factor for increased severity and death in COVID-19 cases.

We concluded that patients having increased blood pressure and body temperature at the time of admission showed a higher risk for severity in COVID-19. It remains unclear whether managing the elevated blood pressure and temperature of critically ill patients with COVID-19 will alleviate the inflammatory response and boost the result. Additional research is required to answer the topic of blood pressure, temperature, and gender. These study limitations are the lack of follow-up of blood pressure and temperature during hospital admission. This study will help in the early detection of the severity and nonseverity of COVID-19 cases, which is very necessary to deal with the current scenario.

\section{ACKNOWLEDGMENTS}

The authors are grateful to Era University for providing the lab space and financial assistance. 
Declaration of Conflicting Interests: The authors declare that they have no conflict of interest.

Funding: Era University in Lucknow, India, financed the research with intramural research grants.

\section{REFERENCES}

1. Li LQ, Huang T, Wang YQ, et al. COVID-19 patients' clinical characteristics, discharge rate, and fatality rate of meta-analysis. J Med Virol.2020;92(6):577-583.

2. CDC. Interim Clinical Guidance for Management of Patients with Confirmed Coronavirus Disease (COVID-19). Available online: https://www.cdc.gov/coronavirus/2019-

$\mathrm{ncov} / \mathrm{hcp} /$ clinical-guidancemanagement-

patients.html (accessed on Aug 28 2020).

3. WHO. Report of the WHO-China Joint Mission on coronavirus disease 2019 (COVID-19). 16-24 Feb., 2020. https://www.who.int/docs/defaultsource/coronaviruse/who-china-joint-mission-oncovid-19-final-report.pdf (accessed March 26, 2020).

4. Liang $\mathrm{W}$, Liang $\mathrm{H}$, Ou L, et al. Development and validation of a clinical risk score to predict the occurrence of critical illness in hospitalized patients with COVID-19. JAMA Intern Med. 2020;180(8): 1081-1089.

5. De Terwangne $C$, Laouni J, Jouffe $L$, et al. Predictive accuracy of COVID-19 world health organization (WHO) severity classification and comparison with a bayesian-method-based severity score (Epi-score). Pathogens. 2020;9(11):880.

6. Grasselli G, Zangrillo A, Zanella A, et al. Baseline characteristics and outcomes of 1591 patients infected with SARS-CoV-2 admitted to ICUs of the Lombardy Region, Italy. Jama. 2020;323(16):1574-1581.

7. Huang $\mathrm{C}$, Wang $\mathrm{Y}$, Li X, et al. Clinical features of patients infected with 2019 novel coronavirus in Wuhan, China. Lancet. 2020;395(10223):497-506.

8. Yang $X, Y u Y, X u ~ J$, et al. Clinical course and outcomes of critically ill patients with SARS-CoV-2 pneumonia in Wuhan, China: a single-centered, retrospective, observational study. Lancet Respir Med. 2020;8(5):475-481

9. Liu K, Fang $Y Y$, Deng $Y$, et al. Clinical characteristics of novel coronavirus cases in tertiary hospitals in Hubei Province. Chin Med J (Engl). 2020 133;(9):1025-1031.

10. Gao C, Cai $Y$, Zhang K, et al. Association of hypertension and antihypertensive treatment with COVID-19 mortality: a retrospective observational study. Eur Heart J. 2020;41(22):2058-2066.

11. Gostic K, Gomez AC, Mummah RO, et al. Estimated effectiveness of symptom and risk screening to prevent the spread of COVID-19. Elife. 2020;24(9):e55570.
12. Ogoina D. Fever, fever patterns and diseases called 'fever'--a review. J Infect Public Health. 2011;4(3):108-24

13. Saladin KS, Miller L. Anatomy and Physiology: The Unity of Form and Function, 6th edn. New York: McGraw-Hill, 2011: 1-27.

14. Plaza JJ, Hulak N, Zhumadilov Z, et al. Fever as an important resource for infectious diseases research. Intractable Rare Dis Res. 2016;5(2):97102.

15. Mitra B, Luckhoff C, Mitchell RD, et al. Temperature screening has negligible value for control of COVID-19. Emerg Med Australas. 2020:32(5):867-869.

16. Liu JY, Chen TJ, Hwang SJ. Analysis of imported cases of COVID-19 in Taiwan: a nationwide study. Int J Environ Res Public Health. 2020;17(9):3311.

17. Lechien JR, Chiesa-Estomba CM, Place S, et al. Clinical and epidemiological characteristics of 1420 European patients with mild-to-moderate coronavirus disease 2019. J Intern Med. 2020;288(3):335-344.

18. Kanwal A, Agarwala A, Martin LW, et al. COVID-19 and Hypertension: What We Know and Don't Know. American College of Cardiology. 2020;6.

19. Zhang $P$, Zhu L, Cai J, et al. Association of Inpatient Use of Angiotensin-Converting Enzyme Inhibitors and Angiotensin II Receptor Blockers With Mortality Among Patients With Hypertension Hospitalized With COVID-19. Circ Res. 2020;126(12):1671-1681.

20. Chen $N$, Zhou $M$, Dong $X$, et al. Epidemiological and clinical characteristics of 99 cases of 2019 novel coronavirus pneumonia in Wuhan, China: a descriptive study. Lancet. 2020;395(10223):507-513.

21. Kulkarni S, Jenner BL, Wilkinson I. COVID-19 and hypertension. J Renin Angiotensin Aldosterone Syst. 2020;21(2):1470320320927851.

22. Tharakan S, Nomoto K, Miyashita S, et al. Body temperature correlates with mortality in COVID-19 patients. Crit Care. 2020;24(1):1-3.

23. Bielecki M, Crameri GA, Schlagenhauf $P$, et al. Body temperature screening to identify SARS-CoV2 infected young adult travellers is ineffective. Travel Med Infect Dis. 2020;37:101832.

24. Li X, Xu S, Yu M, et al. Risk factors for severity and mortality in adult COVID-19 inpatients in Wuhan. J Allergy Clin Immunol. 2020;146(1):110118.

25. Zhang JJ, Dong $X$, Cao $Y Y$, et al. Clinical characteristics of 140 patients infected with SARSCoV-2 in Wuhan, China. Allergy. 2020;75(7):1730-1741.

26. Bai $Y$, Yao $L$, Wei $T$, et al. Presumed asymptomatic carrier transmission of COVID-19. JAMA. 2020;323(14):1406-1407. 